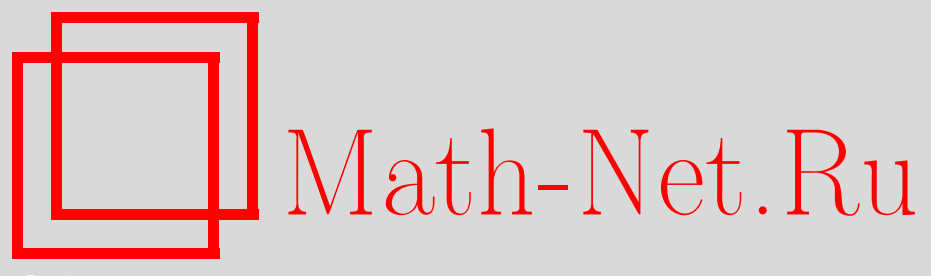

Н. В. Денисова, В. В. Козлов, Д. В. Трещёв, Замечания о полиномиальных интегралах высших степеней обратимых систем с торическим пространством конфигураций, Изв. РАН. Сер. матем., 2012, том 76, выпуск 5, 57-72

DOI: https://doi.org/10.4213/im8001

Использование Общероссийского математического портала Math-Net.Ru подразумевает, что вы прочитали и согласны с пользовательским соглашением http://www . mathnet.ru/rus/agreement

Параметры загрузки:

IP : 3.85 .73 .92

26 апреля 2023 г., 14:46:03

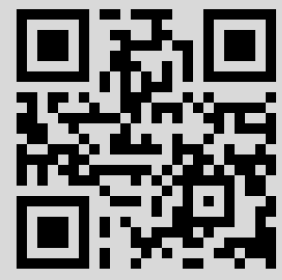


УДК $517.9+531.01$

\author{
Н. В. Денисова, В. В. Козлов, Д. В. Трещёв
}

\title{
Замечания о полиномиальных интегралах высших степеней обратимых систем с торическим пространством конфигураций
}

\begin{abstract}
Рассматриваются вопросы, связанные с известной гипотезой о степени неприводимых полиномиальных интегралов обратимой гамильтоновой системы с двумя степенями свободы и торическим пространством положений. Основное внимание уделено исследованию особой системы, возникающей при анализе неприводимых полиномиальных интегралов четвертой степени. В частном случае имеем задачу о движении двух взаимодействующих частиц по окружности в заданных потенциальных силовых полях. Доказано, что если все три потенциала - гладкие непостоянные функции, то эта задача не допускает нетривиальных полиномиальных интегралов сколь угодно высокой степени. Упомянутая выше гипотеза полностью доказана для систем с полиномиальным по импульсам первым интегралом четвертой степени.

Библиография: 8 наименований.
\end{abstract}

Ключевые слова: неприводимые интегралы, системы с ударами, спектр потенциала.

\section{$\S 1$. Введение}

В настоящей работе рассматривается обратимая механическая система с двумя степенями свободы, динамика которой описывается дифференциальными уравнениями Гамильтона

$$
\dot{x}_{k}=\frac{\partial H}{\partial y_{k}}, \quad \dot{y}_{k}=-\frac{\partial H}{\partial x_{k}}, \quad k=1,2 .
$$

Предполагается, что гамильтониан имеет вид

$$
H=\frac{1}{2}\left(y_{1}^{2}+y_{2}^{2}\right)-W\left(x_{1}, x_{2}\right)
$$

где $W$ - гладкая (бесконечно дифференцируемая) функция на двумерном конфигурационном торе

$$
\mathbb{T}^{2}=\left\{x_{1}, x_{2} \bmod 2 \pi\right\} .
$$

Работа первого автора выполнена при финансовой поддержке РФФИ (гранты № 12-01-00648-a, 11-01-12075-офи-м-2011) и Программы Правительства РФ "Господдержка научных исследований, проводимых под руководством ведущих ученых" в ФГБОУ ВПО Московский государственный университет им. М. В. Ломоносова (грант № 11.G34.31.0054).

Работа третьего автора выполнена при финансовой поддержке Программы Правительства РФ "Господдержка научных исследований, проводимых под руководством ведущих ученых" в ФГБОУ ВПО Московский государственный университет им. М. В. Ломоносова (грант № 11.G34.31.0039). 
Изучается вопрос о наличии нетривиальных первых интегралов уравнений (1.1), которые представляются полиномами по импульсам $y_{1}, y_{2}$ с однозначными на $\mathbb{T}^{2}$ коэффициентами. Нетривиальность означает функциональную независимость таких интегралов от интеграла энергии (1.2). Полиномиальный по импульсам интеграл называется неприводимым, если не существует нетривиального полиномиального интеграла уравнений (1.1) меньшей степени.

Известна следующая гипотеза: в рассматриваемой задаче степень неприводимого полиномиального интеграла не превосходит двух (см. [1]). Данная гипотеза в полном объеме пока не доказана. Обзор результатов в этом направлении, полученных до 2000 г., содержится в работе [2].

Единственный известный нам более поздний результат указан в работе [3], где автор пытается доказать сформулированную выше гипотезу для интегралов степени $n=5$. Однако доказательство, приведенное в [3], неполное. В нем неявно используется то, что одна из прямых спектра потенциала вертикальна или горизонтальна. Поэтому вопрос об интеграле пятой степени следует считать открытым. Вообще, случай нечетного $n$ более простой: здесь степень неприводимого интеграла, по-видимому, равна единице (и поэтому его существование связано с нётеровой группой симметрий). Доказательство гипотезы в случае $n=3$ не представляет большого труда, и оно приведено полностью в [2].

Полиномиальные интегралы четвертой степени также до конца не изучены. Точнее, в [2] доказательство гипотезы о неприводимых интегралах сведено к рассмотрению одного особого случая, что представляет собой не простую задачу. Анализ этой исключительной гамильтоновой системы и составляет содержание настоящей работы.

\section{§ 2. Полиномиальные интегралы четвертой степени. Предварительный анализ}

Приведем явный вид уравнений Гамильтона (1.1):

$$
\dot{x}_{1}=y_{1}, \quad \dot{x}_{2}=y_{2}, \quad \dot{y}_{1}=\frac{\partial W}{\partial x_{1}}, \quad \dot{y}_{2}=\frac{\partial W}{\partial x_{2}} .
$$

Как показано в [2], неприводимый интеграл четвертой степени (если он существует) всегда можно представить в виде

$$
F=\frac{1}{4}\left(y_{1}^{4}+y_{2}^{4}\right)+\frac{a}{2} y_{1}^{2}+b y_{1} y_{2}+\frac{c}{2} y_{2}^{2}+g .
$$

Здесь $a, b, c$ и $g$ - гладкие $2 \pi$-периодические функции относительно $x_{1}$ и $x_{2}$.

Полная производная от функции $F$ в силу гамильтоновой системы $(2.1)$ есть неоднородный многочлен по импульсам степени 3. Полагая равной нулю сумму слагаемых третьей степени, получаем систему уравнений

$$
\begin{array}{ll}
\frac{\partial W}{\partial x_{1}}+\frac{1}{2} \frac{\partial a}{\partial x_{1}}=0, & \frac{1}{2} \frac{\partial a}{\partial x_{2}}+\frac{\partial b}{\partial x_{1}}=0 \\
\frac{\partial W}{\partial x_{2}}+\frac{1}{2} \frac{\partial c}{\partial x_{2}}=0, & \frac{1}{2} \frac{\partial c}{\partial x_{1}}+\frac{\partial b}{\partial x_{2}}=0 .
\end{array}
$$


Приравнивая нулю коэффициенты при $y_{1}$ и $y_{2}$, получаем уравнения

$$
a \frac{\partial W}{\partial x_{1}}+b \frac{\partial W}{\partial x_{2}}+\frac{\partial g}{\partial x_{1}}=0, \quad b \frac{\partial W}{\partial x_{1}}+c \frac{\partial W}{\partial x_{2}}+\frac{\partial g}{\partial x_{2}}=0 .
$$

Соотношения (2.2)-(2.4) составляют полную совокупность условий инвариантности функции $F$ относительно фазового потока исходной гамильтоновой системы.

Из первых уравнений в (2.2) и (2.3) получаем

$$
a=-2 W+2 A\left(x_{2}\right), \quad c=-2 W+2 C\left(x_{1}\right) .
$$

Здесь $A$ и $C$ - пока неизвестные гладкие $2 \pi$-периодические функции одной переменной. Вторые уравнения в (2.2) и (2.3) вместе с (2.5) дают нам соотношения

$$
\frac{\partial b}{\partial x_{1}}=\frac{\partial W}{\partial x_{2}}-A^{\prime}, \quad \frac{\partial b}{\partial x_{2}}=\frac{\partial W}{\partial x_{1}}-C^{\prime} .
$$

Отсюда следует, что функция $b$ удовлетворяет гиперболическому дифференциальному уравнению

$$
\frac{\partial^{2} b}{\partial x_{1}^{2}}-\frac{\partial^{2} b}{\partial x_{2}^{2}}=0
$$

Значит,

$$
b=B\left(x_{1}+x_{2}\right)-D\left(x_{1}-x_{2}\right),
$$

где $B$ и $D$ - гладкие $2 \pi$-периодические функции одной переменной. Поскольку

$$
\frac{\partial B}{\partial x_{1}}=\frac{\partial B}{\partial x_{2}}, \quad \frac{\partial D}{\partial x_{1}}=-\frac{\partial D}{\partial x_{2}},
$$

из (2.6) и (2.7) вытекает, что с точностью до несущественного аддитивного слагаемого потенциальная энергия гамильтоновой системы имеет вид

$$
W=A\left(x_{2}\right)+C\left(x_{1}\right)+B\left(x_{1}+x_{2}\right)+D\left(x_{1}-x_{2}\right) .
$$

Преобразуем теперь систему (2.4) с учетом формул (2.5) и (2.7):

$$
\begin{aligned}
& 2 A \frac{\partial W}{\partial x_{1}}+(B-D) \frac{\partial W}{\partial x_{2}}+\frac{\partial T}{\partial x_{1}}=0, \\
& (B-D) \frac{\partial W}{\partial x_{1}}+2 C \frac{\partial W}{\partial x_{2}}+\frac{\partial T}{\partial x_{2}}=0,
\end{aligned}
$$

где $T=g-W^{2}$. Исключая функцию $T$, приходим к соотношению, связывающему функции $A, B, C, D$ из (2.8):

$$
\begin{aligned}
3 A^{\prime}\left(B^{\prime}+D^{\prime}\right)+2 A\left(B^{\prime \prime}\right. & \left.-D^{\prime \prime}\right)+A^{\prime \prime}(B-D) \\
& =3 C^{\prime}\left(B^{\prime}-D^{\prime}\right)+2 C\left(B^{\prime \prime}-D^{\prime \prime}\right)+C^{\prime \prime}(B-D) .
\end{aligned}
$$

Штрих обозначает производную функции одной переменной по своему аргументу. В частности,

$$
D^{\prime}=-\frac{\partial D}{\partial x_{2}}
$$


Соотношение (2.10) является ключевым для дальнейшего анализа. Это уравнение получено также в [2], однако в [2] оно содержит еще дополнительные слагаемые, пропорциональные некоторым константам $c_{1}$ и $c_{2}$. Легко показать, что на самом деле $c_{1}$ и $c_{2}$ равны нулю.

Отметим, что уравнение (2.10) получено раньше в работе [4] в другом контексте. В [4] рассматривалась задача о полиномиальных интегралах четвертой степени для уравнения (2.1) с потенциальной энергией вида (2.8). Другими словами, вид потенциала уже задан и его периодичность не предполагается. Как показано в [4], частным решением (2.10) являются §-функция Вейерштрасса и функция ее вырождения. Конечно, §-функция периодична, но имеет сингулярности на вещественной оси (полюсы второго порядка). Наша задача совсем иная: доказать, что (2.10) имеет только тривиальные гладкие периодические решения (либо $A=C=0$, либо $B=D=0$ ).

\section{§ 3. Анализ Фурье}

Для исследования нелинейного уравнения (2.10) применим метод Фурье. Пусть

$$
\begin{aligned}
A(z) & =\sum_{n \in \mathbb{Z}} A_{n} e^{i n z}, & C(z) & =\sum_{n \in \mathbb{Z}} C_{n} e^{i n z}, \\
B(z) & =\sum_{n \in \mathbb{Z}} B_{n} e^{i n z}, & D(z) & =\sum_{n \in \mathbb{Z}} D_{n} e^{i n z}
\end{aligned}
$$

- разложения Фурье периодических слагаемых в (2.8). Поскольку потенциальная энергия определяется с точностью до произвольной аддитивной постоянной, можно считать вещественные числа $A_{0}, B_{0}, C_{0}$ и $D_{0}$ равными нулю.

Подставляя разложения (3.1) в (2.10) и группируя коэффициенты Фурье при одной и той же гармонике $e^{i\left(\alpha x_{1}+\beta x_{2}\right)}, \alpha, \beta \in \mathbb{Z}$, получим следующее алгебраическое соотношение:

$$
\begin{aligned}
& \beta(\alpha+\beta) A_{\beta-\alpha} B_{\alpha}+\beta(\alpha-\beta) A_{\alpha+\beta} D_{\alpha} \\
& =\alpha(\alpha+\beta) C_{\alpha-\beta} B_{\beta}+\alpha(\beta-\alpha) C_{\alpha+\beta} D_{-\beta} .
\end{aligned}
$$

Если числа $\alpha, \beta, \alpha-\beta, \alpha+\beta$ не равны нулю, то (3.2) можно переписать следующим образом:

$$
a_{\beta-\alpha} b_{\alpha}-a_{\alpha+\beta} d_{\alpha}+c_{\alpha-\beta} b_{\beta}+c_{\alpha+\beta} d_{-\beta}=0 .
$$

Здесь для краткости записи введены обозначения:

$$
a_{p}=\frac{A_{p}}{p}, \ldots, d_{q}=\frac{D_{q}}{q}, \quad p \neq 0, \ldots, q \neq 0 .
$$

Числа $a_{p}, \ldots, d_{q}-$ коэффициенты Фурье первообразных периодических функций $A, \ldots, D$ одной переменной. При $p=0, \ldots, q=0$ эти коэффициенты не определены (поскольку по нашему соглашению $A_{0}=C_{0}=B_{0}=D_{0}=0$ ). Отметим, что соотношения (3.3) справедливы, только если все целые числа $\alpha$, $\beta, \alpha-\beta$ и $\alpha+\beta$ отличны от нуля. 


\section{$\S 4$. Сингулярные решения}

Цепочка алгебраических уравнений (3.2) имеет нетривиальные решения. Укажем одно из них, полагая $B(z)=0$. В частности, в $(3.2)$ все $B_{\alpha}$ равны нулю. Далее, пусть $D$ - четная функция (возможно, обобщенная) и

$$
D_{\alpha}=\alpha d, \quad d \in \mathbb{R} \backslash\{0\}
$$

Ясно, что $d_{\alpha}=d$ - коэффициенты Фурье периодической обобщенной функции

$$
2 \pi d \delta(z), \quad z \bmod 2 \pi,
$$

a $-i D_{\alpha}-$ коэффициенты Фурье ее производной. Периодическая $\delta$-функция Дирака с периодом $2 \pi$ определяется расходящимся рядом Фурье

$$
\frac{1}{2 \pi} \sum_{n=-\infty}^{+\infty} e^{i n z}, \quad z \in \mathbb{R} .
$$

Чтобы не менять среднего значения этой обобщенной функции, коэффициент $d_{0}$ в (4.1) следует положить равным остальным коэффициентам $d_{\alpha}, \alpha \neq 0$, которые совпадают между собой согласно предположению.

Если подставить выражение (4.2) в формулу

$$
\int_{0}^{2 \pi} f(x) \delta(x) d x
$$

и формально проинтегрировать, то получим $\sum f_{n}$, где $f_{n}, n \in \mathbb{Z},-$ коэффициенты Фурье гладкой функции $f$. Ясно, что сумма этого ряда есть $f(0)=f(2 \pi)$. С другой стороны, ряд (4.2) представляется тригонометрическим рядом только по косинусам:

$$
\frac{1}{2 \pi}+\frac{1}{\pi} \sum_{n=1}^{\infty} \cos (n z) .
$$

Отметим, что при $0<z<2 \pi$ этот ряд сходится по Чезаро к нулю (см. [5, п. 1.2]), а при $z=0(z=2 \pi)$ его сумма, очевидно, равна $+\infty$. Эти замечания вполне согласуются с интуитивным определением периодической $\delta$-функции Дирака.

Учитывая предположение (4.1), из (3.2) получаем равенство

$$
A_{\alpha+\beta}=C_{\alpha+\beta}
$$

Итак, уравнение (2.10) допускает частное решение

$$
A(z)=C(z), \quad B=0, \quad D(z)=\lambda \delta^{\prime}(z), \quad \lambda=\text { const } .
$$

В этом случае исходные дифференциальные уравнения Гамильтона допускают полиномиальный по импульсам интеграл четвертой степени. Если $\lambda \neq 0$ и функция $A$ (или $C$ ) не постоянна, то этот интеграл будет неприводимым. 


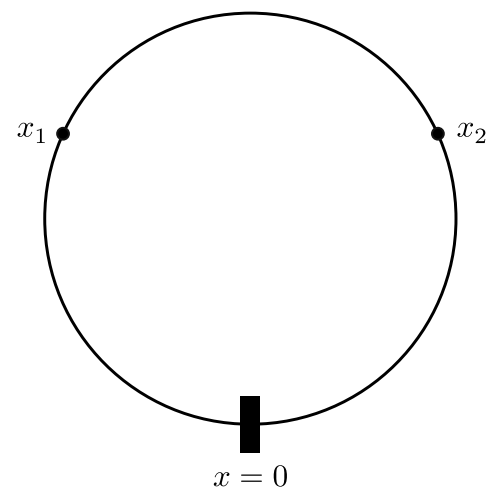

Рис. 1. Динамика двух одинаковых частиц

Отметим, что решение (4.3), действительно, является обобщенным решением уравнения (2.10). Это означает следующее. Умножая уравнение (2.10) на произвольную "пробную" гладкую функцию $\varphi: \mathbb{T}^{2} \rightarrow \mathbb{R}$ и производя формальные интегрирования по частям, после подстановки (4.3) получаем равенство.

Этому результату можно дать следующую содержательную интерпретацию в терминах систем с упругими ударами (биллиардов). Рассмотрим динамику двух одинаковых частиц, движущихся по окружности $\{x \bmod 2 \pi\}$. Пусть $x_{1}$ и $x_{2}$ - их координаты. Предположим, что частицы могут упруго (без потери энергии) соударяться. Потенциальная энергия их парного взаимодействия пропорциональна производной $2 \pi$-периодической дельта-функции Дирака. Далее, будем считать, что эти частицы упруго сталкиваются со стенкой $x=0$ $(x=2 \pi)$ с обеих сторон (рис. 1). Другими словами, на каждую из частиц действует обобщенная сила, которая также выражается через дельта-функцию Дирака (в частности, выполнено равенство $A=C$ ). Кроме интеграла энергии $\left(y_{1}^{2}+y_{2}^{2}\right) / 2$, эта система допускает неприводимый первый интеграл

$$
y_{1}^{4}+y_{2}^{4}
$$

Действительно, при движении между ударами импульсы частищ $y_{1}$ и $y_{2}$ сохраняются. При ударах о неподвижную преграду их импульсы меняют знак, а при упругом соударении между собой частицы меняются импульсами. Поэтому (4.4), конечно, будет первым интегралом. В качестве интеграла четвертой степени можно также принять $y_{1}^{2} y_{2}^{2}$. Эта симметрическая функция выражается через интеграл (4.4) и кинетическую энергию системы частиц. Нетрудно показать, что интеграл (4.4) будет неприводимым (т. е. данная система не имеет полиномиальных интегралов степени не выше 3, не зависимых от интеграла энергии). В доказательстве используются два факта:

a) интеграл не зависит от $x_{1}$ и $x_{2}$;

b) он не меняется при подстановках

$$
y_{1} \mapsto-y_{1}, \quad y_{2} \mapsto-y_{2} ; \quad y_{1} \mapsto y_{2}, \quad y_{2} \mapsto y_{1}
$$


ЗАмечАниЕ 4.1. Пусть $D_{\alpha}-$ коэффициенты Фурье обобщенной функции, которые линейно растут с номером $\alpha$. Тогда ее тригонометрический ряд

$$
\lambda \sum_{n=-\infty}^{+\infty} n e^{i n z}=2 \lambda \sum_{n=1}^{\infty} n \cos (n z), \quad \lambda=\mathrm{const}
$$

при $0<z<2 \pi$ суммируется методом Чезаро (C, 2) (см. [5]) к функции

$$
D(z)=-\frac{\lambda}{2 \sin ^{2} \frac{z}{2}} .
$$

Это одно из вырождений $९$-функции Вейерштрасса. Хорошо известно, что гамильтонова система с потенциалом

$$
V=D\left(x_{1}\right)+D\left(x_{2}\right)+D\left(x_{1}-x_{2}\right)+D\left(x_{1}+x_{2}\right)
$$

допускает неприводимый интеграл четвертой степени по импульсам [4].

\section{§5. Суммируемые решения для двух взаимодействующих частиц}

Рассмотрим вопрос о существовании решений уравнения (2.10), коэффициенты Фурье которых стремятся к нулю при стремлении их номеров к бесконечности. Несколько злоупотребляя терминологией, мы будем называть такие решения суммируемыми. Сначала мы также рассмотрим частный случай, когда $B=0$. Как и в $\S 4$, этот случай допускает прозрачную механическую интерпретацию: движение двух взаимодействующих частиц по окружности, каждая из которых находится в потенциальном силовом поле.

Теорема 5.1. Пусть $B=0 u d_{n} \rightarrow 0$ при $|n| \rightarrow \infty$. Если исходная гамильтонова система имеет полиномиальный по импульсам интеграл четвертой степени, то существует нетривиальный полиномиальный интеграл степени не выие 2.

ДокАЗАТЕЛЬСТво. При $B=0$ цепочка уравнений (3.3) упрощается:

$$
a_{\alpha+\beta} d_{\alpha}=c_{\alpha+\beta} d_{-\beta} .
$$

Теперь поменяем местами $\alpha$ и $\beta$ :

$$
a_{\alpha+\beta} d_{\beta}=c_{\alpha+\beta} d_{-\alpha} .
$$

Соотношения (5.1) и (5.2) составляют линейную систему уравнений относительно $a_{\alpha+\beta}$ и $c_{\alpha+\beta}$. Если хотя бы одно из этих чисел не равно нулю, то определитель системы обращается в нуль:

$$
\left|d_{\alpha}\right|^{2}-\left|d_{\beta}\right|^{2}=0
$$

В рассматриваемой ситуации можно считать, что хотя бы одна из функций $A$ или $C$ не тождественно равна нулю. В противном случае спектр потенциала $W$ лежит на двух прямых, но тогда заключение теоремы вытекает из общих результатов работы [6]. 
Итак, пусть, например, $A_{p} \neq 0$ при некотором $p \neq 0$. Тогда $a_{p} \neq 0$ и для бесконечного количества наборов целых чисел $\alpha, \beta$ коэффициенты Фурье $a_{\alpha+\beta}$ отличны от нуля (надо просто положить $\beta=p-\alpha$ ). Пусть $d_{\alpha} \neq 0$ при некотором $\alpha \neq 0$. В противном случае $D \equiv 0$ и уравнения Гамильтона заведомо допускают квадратичный интеграл ввиду разделения переменных $x_{1}, y_{1}$ и $x_{2}, y_{2}$. Тогда из (5.3) вытекает цепочка равенств

$$
\begin{aligned}
\left|d_{\alpha}\right|=\left|d_{-\alpha}\right|=\left|d_{\alpha+p}\right|=\left|d_{-\alpha-p}\right| & =\left|d_{\alpha+2 p}\right| \\
& =\left|d_{-\alpha-2 p}\right|=\left|d_{\alpha+3 p}\right|=\left|d_{-\alpha-3 p}\right|=\cdots .
\end{aligned}
$$

Поскольку $d_{\alpha} \neq 0$, последовательность $d_{\alpha+m p}, m=0,1,2, \ldots$, не стремится к нулю при $m \rightarrow \infty$, но это противоречит предположению теоремы.

\section{§ 6. Отсутствие полиномиальных интегралов высших степеней}

Теорему 5.1 можно усилить. Для простоты функции $A, B, C$ и $D$ считаем гладкими.

Теорема 6.1. Пусть $B=0$ в формуле (2.8), а функиии $A$ (или $C$ ) и $D$ непостоянные. Тогда уравнения (2.1) не допускают нетривиального полиномиального по импульсам первого интеграла.

ДокАзАТЕЛьство основано на других идеях. Оно использует теорию возмущений и основано на применении теоремы $1_{2}$ из [7, гл. IV].

Пусть

$$
W=\sum w_{\lambda} e^{i(\lambda, x)}, \quad \lambda \in \mathbb{Z}^{2},
$$

- ряд Фурье потенциальной энергии. Ясно, что

$$
w_{0, \beta}=A_{\beta}, \quad w_{\beta, 0}=C_{\beta}, \quad w_{\alpha, \alpha}=B_{\alpha}, \quad w_{\alpha,-\alpha}=D_{\alpha} .
$$

Множество Пуанкаре $P_{1}$ первого порядка - это четыре резонансные прямые на плоскости импульсов $\mathbb{R}^{2}=\left\{y_{1}, y_{2}\right\}$ :

$$
y_{1}=0, \quad y_{2}=0, \quad y_{1}+y_{2}=0, \quad y_{1}-y_{2}=0 .
$$

Их число уменьшается, если одна из функций $A, \ldots, D$ обращается в тождественный нуль.

Следуя [7], рассмотрим суммы

$$
\widetilde{w}_{k}=\frac{1}{2} \sum_{\tau+\sigma=k} \frac{(\tau, \sigma) w_{\tau} w_{\sigma}}{(y, \tau)(y, \sigma)}, \quad k=(\alpha, \beta) \neq 0,
$$

и определим множество Пуанкаре $P_{2}$ второго порядка как совокупность прямых

$$
(k, y)=\alpha y_{1}+\beta y_{2}=0,
$$

на которых соответствующие функции $\widetilde{w}_{k}$ не равны нулю.

Согласно теореме $1_{2}$, если множество $P_{2}$ состоит из бесконечного числа различных прямых, исходная гамильтонова система не допускает дополнительного 
полиномиального по импульсам первого интеграла. Легко проверить, что прямая (6.1) не принадлежит $P_{2}$, если выполнено условие (3.3). Другими словами, $\widetilde{w}_{k}=0$ на прямой $(6.1)$ в том и только в том случае, когда выполнено условие (3.3). Отметим совпадение этих условий, хотя они получены из разных предположений и поэтому имеют разный смысл.

Теперь мы снова полагаем $B=0$. Целочисленный вектор $(\alpha, \beta) \neq 0$ задает прямую (6.1) из множества Пуанкаре второго порядка, если

$$
a_{\alpha+\beta} d_{\alpha}-c_{\alpha+\beta} d_{-\beta} \neq 0
$$

Если таких различных прямых бесконечно много, то теорема доказана. Пусть теперь $P_{2}$ (как и $P_{1}$ ) состоит из конечного числа прямых. Тогда мы имеем только конечное число различных неравенств вида (6.2).

Рассмотрим целочисленные векторы $(\alpha, \beta)$ такие, что:

1) $\alpha+\beta=p=$ const;

2) $a_{\alpha+\beta}$ или $c_{\alpha+\beta}$ отлично от нуля;

3) прямые $\alpha y_{1}+\beta y_{2}=0$ и $\beta y_{1}+\alpha y_{2}=0$ не принадлежат множеству $P_{2}$.

Рассуждая так же, как и при доказательстве теоремы 5.1, приходим к соотношению

$$
\left|d_{\alpha}\right|=\left|d_{\beta}\right|, \quad \beta=p-\alpha
$$

Отсюда вытекает, что функция $D(\cdot)$ - тригонометрический многочлен (т. е. $d_{\alpha}=0$ при всех $\left.|\alpha| \geqslant \alpha_{0}\right)$. Действительно, поскольку множество $P_{2}$ состоит из конечного числа прямых, из (6.3) вытекает цепочка равенств (5.4), справедливых при достаточно больших значениях $\alpha$. Однако тогда из (5.4) вытекает, что коэффициенты Фурье первообразной функции $z \mapsto D(z)$ не стремятся к нулю при увеличении их номеров.

Итак, согласно (6.3), если одна из функций $A$ или $C$ не равна тождественно нулю, найдутся по крайней мере два целых числа $0<\beta<\alpha$ таких, что $d_{\alpha} \neq 0$ и $d_{\beta} \neq 0$.

Докажем, что функции $A$ и $C$ являются также тригонометрическими многочленами. Пусть это не так. Тогда найдется возрастающая последовательность целых чисел $p_{1}, p_{2}, \ldots$, стремящаяся к бесконечности и такая, что $a_{p_{m}} \neq 0$ (случай $c_{p_{m}} \neq 0$ рассматривается аналогично). Количество пересечений прямых $\alpha=$ const (и $\beta=$ const) и $\alpha+\beta=p_{m}$, очевидно, бесконечно.

Пусть $\alpha$ - наибольшее из целых неотрицательных чисел такое, что $d_{\alpha} \neq 0$. При больших $m$, очевидно,

$$
\beta=p_{m}-\alpha>\alpha
$$

Однако тогда, согласно (6.3) имеем $\left|d_{-\beta}\right|=\left|d_{\beta}\right| \neq 0$. Противоречие.

Таким образом, потенциальная энергия $W$ - тригонометрический многочлен, а в этом случае справедливость теоремы вытекает из общего результата работы $[6]$. 


\section{§ 7. Суммируемые решения в общей задаче}

ТеОрема 7.1. Пусть $\left(A_{p}, B_{p}, C_{p}, D_{p}\right), p \in \mathbb{Z},-$ решение цепочки алгебраических уравнений (3.2), причем

$$
\frac{A_{p}}{p}, \quad \frac{B_{p}}{p}, \quad \frac{C_{p}}{p}, \quad \frac{D_{p}}{p}
$$

стремятся к нулю при $|p| \rightarrow \infty$. Если

$$
\sum^{\prime}\left|A_{p}\right| \neq 0 \quad \text { или } \quad \sum^{\prime}\left|C_{p}\right| \neq 0,
$$

где через $\sum^{\prime}$ обозначена сумма по ненулевым иелым индексам $p$, то

$$
\left|B_{p}\right|=\left|D_{p}\right|
$$

при всех цельх р. Наоборот, если

$$
\sum^{\prime}\left|B_{p}\right| \neq 0 \quad \text { или } \quad \sum^{\prime}\left|D_{p}\right| \neq 0
$$

mo

$$
\left|A_{p}\right|=\left|C_{p}\right|
$$

при всех иельх р.

СлЕДСТвИЕ. Если одновременно выполнены условия (7.2) и (7.4), то справедливы равенства (7.3) и (7.5) для коэффициентов Фуръе функиий $A, B$, C $u$ D.

Рассмотрим частный случай, когда $B \equiv 0$, а функция $A$ (или $C$ ) не обращается тождественно в нуль. Тогда $D_{p}=0$ при всех $p$ согласно (7.3). Следовательно, $D \equiv 0$, и поэтому исходная гамильтонова система допускает дополнительный квадратичный интеграл. Таким образом, теорема 5.1 по существу содержится в теореме 7.1.

Заметим, что условие теоремы 7.1 о стремлении к нулю последовательностей (7.1) существенно. Действительно, согласно [8] уравнение (2.10) допускает частное решение

$$
A(z)=\frac{a}{(\cos z)^{2}}, \quad C(z)=\frac{c}{(\cos z)^{2}}, \quad-B(z)=D(z)=\cos z,
$$

где $a$ и $c$ - произвольные вещественные постоянные. Ряды Фурье периодических функций $A$ и $C$ (понимаемые в обобщенном смысле) легко получаются из формул (4.5) и (4.6). Конечно, при $a \neq c$ соотношения (7.5) не выполняются. Однако здесь нет противоречия, поскольку коэффициенты Фурье $A_{p}$ и $C_{p}$ линейно растут вместе с номером $p$.

ДОКАЗАТЕЛЬСТВо ТЕОРЕМЫ 7.1 основано на использовании соотношения (3.2) для коэффициентов Фурье четырех гладких функций $A, B, C$ и $D$. Это соотношение справедливо при всех целых $\alpha$ и $\beta$. Делая подстановки

$$
\begin{aligned}
& (\alpha, \beta) \mapsto(-\alpha, \beta), \\
& (\alpha, \beta) \mapsto(\alpha,-\beta), \quad(\alpha, \beta) \mapsto(-\alpha,-\beta), \quad(\alpha, \beta) \mapsto(\beta, \alpha), \\
& (\alpha, \beta) \mapsto(-\beta, \alpha), \quad(\alpha, \beta) \mapsto(\beta,-\alpha), \quad(\alpha, \beta) \mapsto(-\beta,-\alpha),
\end{aligned}
$$




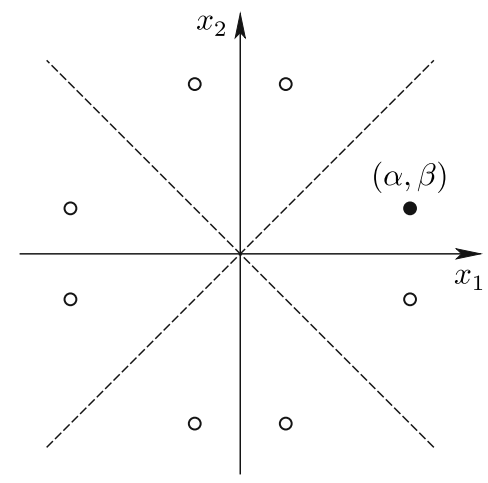

Рис. 2. Образы точки $(\alpha, \beta)$

из (3.2) получим еще семь билинейных соотношений

$$
\begin{gathered}
b_{-\alpha} a_{\alpha+\beta}-d_{-\alpha} a_{-\alpha+\beta}+b_{\beta} c_{-\alpha-\beta}+d_{-\beta} c_{-\alpha+\beta}=0, \\
b_{\alpha} a_{-\alpha-\beta}-d_{\alpha} a_{\alpha-\beta}+b_{-\beta} c_{\alpha+\beta}+d_{\beta} c_{\alpha-\beta}=0, \\
b_{-\alpha} a_{\alpha-\beta}-d_{-\alpha} a_{-\alpha-\beta}+b_{-\beta} c_{-\alpha+\beta}+d_{\beta} c_{-\alpha-\beta}=0, \\
b_{\beta} a_{\alpha-\beta}-d_{\beta} a_{\alpha+\beta}+b_{\alpha} c_{-\alpha+\beta}+d_{-\alpha} c_{\alpha+\beta}=0, \\
b_{-\beta} a_{\alpha+\beta}-d_{-\beta} a_{\alpha-\beta}+b_{\alpha} c_{-\alpha-\beta}+d_{-\alpha} c_{\alpha-\beta}=0, \\
b_{\beta} a_{-\alpha-\beta}-d_{\beta} a_{-\alpha+\beta}+b_{-\alpha} c_{\alpha+\beta}+d_{\alpha} c_{-\alpha+\beta}=0, \\
b_{-\beta} a_{-\alpha+\beta}-d_{-\beta} a_{-\alpha-\beta}+b_{-\alpha} c_{\alpha-\beta}+d_{\alpha} c_{-\alpha-\beta}=0 .
\end{gathered}
$$

На рис. 2 изображены образы точки с целыми координатами $\alpha, \beta$ при подстановках (7.6) (они обозначены кружочками). Это множество из восьми точек симметрично относительно горизонтальной и вертикальной осей, а также относительно биссектрис.

Восемь уравнений (3.3), (7.7) можно рассматривать как линейную однородную алгебраическую систему относительно восьми переменных

$$
b_{\alpha}, b_{\beta}, b_{-\alpha}, b_{-\beta}, d_{\alpha}, d_{\beta}, d_{-\alpha}, d_{-\beta}
$$

либо как линейную систему относительно других восьми величин

$$
a_{\alpha-\beta}, a_{\alpha+\beta}, a_{-\alpha+\beta}, a_{-\alpha-\beta}, c_{\alpha-\beta}, c_{\alpha+\beta}, c_{-\alpha+\beta}, c_{-\alpha-\beta} .
$$

Ввиду вещественности потенциальной энергии $W$ переменные (7.8) (как и величины (7.9)) разбиваются на пары комплексно-сопряженных величин.

Рассмотрим сначала случай, когда среди чисел (7.9) хотя бы одно отлично от нуля. Тогда определитель линейной системы (3.3), (7.7) (относительно переменных (7.9)) будет равен нулю. Этот определитель равен

$$
-\left(b_{\alpha} b_{-\alpha}-d_{\alpha} d_{-\alpha}-b_{\beta} b_{-\beta}+d_{\beta} d_{-\beta}\right)^{4}
$$


Следовательно, если $\alpha \neq 0$ и $\beta \neq 0$, то

$$
\left|b_{\alpha}\right|^{2}-\left|d_{\alpha}\right|^{2}=\left|b_{\beta}\right|^{2}-\left|d_{\beta}\right|^{2} .
$$

Покажем, что

$$
\left|b_{\alpha}\right|=\left|d_{\alpha}\right|
$$

при всех целых $\alpha(\neq 0)$.

Пусть $A_{\alpha-\beta}$ или $C_{\alpha-\beta}$ отличны от нуля (случай, когда $A_{\alpha+\beta}$ или $C_{\alpha+\beta}$ отличны от нуля, рассматривается аналогично). Положим $\alpha-\beta=p$ (или, что то же самое, $\beta=\alpha-p)$. Тогда согласно (7.11) имеем

$$
\left|b_{\alpha}\right|^{2}-\left|d_{\alpha}\right|^{2}=\left|b_{\alpha-p}\right|^{2}-\left|d_{\alpha-p}\right|^{2}
$$

для всех целых $\alpha$. Следовательно,

$$
\left|b_{\alpha}\right|^{2}-\left|d_{\alpha}\right|^{2}=\varkappa=\text { const }
$$

когда $\alpha$ пробегает последовательность целых чисел, кратных $p \neq 0$. Однако тогда $\varkappa=0$, поскольку согласно предположению

$$
b_{\alpha}, d_{\alpha} \rightarrow 0
$$

при $|\alpha| \rightarrow \infty$.

Итак, если периодические функции $A$ или $C$ не равны тождественно нулю, то справедливы равенства (7.12). Точно так же доказываем, что

$$
\left|a_{p}\right|=\left|c_{p}\right|
$$

при всех целых $p(\neq 0)$.

\section{§ 8. Доказательство гипотезы о степени неприводимого интеграла для гладких потенциалов}

Решение $(A, B, C, D)$ функционально-дифференциального уравнения $(2.10)$ назовем тривиальным, если одна из пар функций $A, C$ или $B, D$ - тождественные постоянные.

Теорема 8.1. Уравнение (2.10) не имеет нетривиалъного $C^{3}$-гладкого решения.

С учетом результатов работы [2] отсюда вытекает

СлеДСтвиЕ. Если гамильтонова система (1.1), (1.2) с гладким потенииалом $W$ допускает нетривиальный полиномиальный по импульсам первый интеграл четвертой степени, то найдется нетривиальный полиномиальный интеграл с однозначными на $\mathbb{T}^{2}$ коэффициентами, степень которого не выше 2. 
ДоКАЗАТЕЛЬСтво тЕОРЕмы 8.1. При доказательстве мы развиваем метод работы [2], примененный для изучения полиномиальных интегралов третьей степени.

Полагая $\partial_{1}=\partial / \partial x_{1}$ и $\partial_{2}=\partial / \partial x_{2}$, запишем уравнение $(2.10)$ в виде

$$
\begin{aligned}
\partial_{2}\left(\partial_{1}\right. & \left.+\partial_{2}\right)\left(A\left(x_{2}\right) B\left(x_{1}+x_{2}\right)\right)+\partial_{2}\left(\partial_{1}-\partial_{2}\right)\left(A\left(x_{2}\right) D\left(x_{1}-x_{2}\right)\right) \\
& =\partial_{1}\left(\partial_{1}+\partial_{2}\right)\left(C\left(x_{1}\right) B\left(x_{1}+x_{2}\right)\right)-\partial_{1}\left(\partial_{1}-\partial_{2}\right)\left(C\left(x_{1}\right) D\left(x_{1}-x_{2}\right)\right) .
\end{aligned}
$$

Мы не будем предполагать априори, что средние значения периодических функций $A, B, C, D$ равны нулю. Заметим, что для любого решения $(A(x)$, $B(x), C(x), D(x))$ и для любых постоянных $c_{1}, c_{2}, c_{3}, c_{4} \in \mathbb{R}$ функции

$$
A\left(x+c_{1}\right)-c_{3}, \quad B\left(x+c_{1}+c_{2}\right)-c_{4}, \quad C\left(x+c_{2}\right)-c_{3}, \quad D\left(x+c_{1}-c_{2}\right)-c_{4}
$$

также дают решение уравнения (2.10). С учетом этого замечания далее мы считаем, что

$$
A_{0}+C_{0}=B_{0}+D_{0}=0,
$$

где $A_{0}, B_{0}, C_{0}, D_{0}$ - средние значения функций $A, B, C, D$.

Поскольку

$$
\begin{gathered}
\partial_{2}^{2}\left(A\left(x_{2}\right)\left(B\left(x_{1}+x_{2}\right)-D\left(x_{1}-x_{2}\right)\right)\right)=\partial_{1} \Phi, \\
\Phi=-\partial_{2}\left(A\left(x_{2}\right)\left(B\left(x_{1}+x_{2}\right)+D\left(x_{1}-x_{2}\right)\right)\right) \\
+\left(\partial_{1}+\partial_{2}\right)\left(C\left(x_{1}\right) B\left(x_{1}+x_{2}\right)\right)+\left(\partial_{1}-\partial_{2}\right)\left(C\left(x_{2}\right) D\left(x_{1}-x_{2}\right)\right),
\end{gathered}
$$

усредняя по $x_{1}$, получаем $A^{\prime \prime}(x)\left(B_{0}-D_{0}\right)=0$. Аналогично,

$$
C^{\prime \prime}(x)\left(B_{0}-D_{0}\right)=0, \quad B^{\prime \prime}(x)\left(A_{0}-C_{0}\right)=0, \quad D^{\prime \prime}(x)\left(A_{0}-C_{0}\right)=0 .
$$

Поскольку нас интересуют нетривиальные решения, одна из функций $A, C$ и одна из функций $B, D$ не являются тождественными постоянными. Следовательно, получаем, что $A_{0}=C_{0}$ и $B_{0}=D_{0}$. С учетом (8.2) имеем

$$
A_{0}=C_{0}=B_{0}=D_{0}=0 \text {. }
$$

Далее путем выбора постоянных $c_{1}, c_{2}$ мы будем производить "нормализацию” решений, сдвигая в нуль критические точки. Положим

$$
q_{ \pm}^{\prime}(x)=B(x)-D( \pm x), \quad p_{ \pm}^{\prime}(x)=A\left( \pm \frac{x}{2}\right)-C\left(\frac{x}{2}\right) .
$$

Средние от периодических функций $q_{ \pm}, p_{ \pm}$пока не фиксируются и далее выступают в качестве постоянных интегрирования.

ПРЕДЛОЖЕНИЕ 8.1. Любое решение уравнения (2.10) удовлетворяет следующим четырем свойствам:

1) при условии $q_{+} \not \equiv 0$ и нормализачии $A^{\prime}(0)=0$

$$
\begin{gathered}
C=a_{0}+\frac{a_{2} q_{+}^{2}-\lambda_{+}}{2\left(q_{+}^{\prime}\right)^{2}}, \\
\lambda_{+}=\text {const, } \quad a_{0}=A(0), \quad a_{2}=A^{\prime \prime}(0) ;
\end{gathered}
$$


2) при условии $q_{-} \not \equiv 0$ и нормализации $C^{\prime}(0)=0$

$$
A=c_{0}+\frac{c_{2} q_{-}^{2}-\lambda_{-}}{2\left(q_{-}^{\prime}\right)^{2}}, \quad \lambda_{-}=\mathrm{const}, \quad c_{0}=C(0), \quad c_{2}=C^{\prime \prime}(0)
$$

3) при условии $p_{+} \not \equiv 0$ и нормализащии $D^{\prime}(0)=0$

$$
B=d_{0}+\frac{d_{2} p_{+}^{2}-\mu_{+}}{2\left(p_{+}^{\prime}\right)^{2}}, \quad \mu_{+}=\text {const }, \quad d_{0}=D(0), \quad d_{2}=D^{\prime \prime}(0)
$$

4) при условии $p_{-} \not \equiv 0$ и нормализащии $B^{\prime}(0)=0$

$$
D=b_{0}+\frac{b_{2} p_{-}^{2}-\mu_{-}}{2\left(p_{-}^{\prime}\right)^{2}}, \quad \mu_{-}=\text {const }, \quad b_{0}=B(0), \quad b_{2}=B^{\prime \prime}(0) .
$$

ДокАЗАТЕЛЬСтво. Проверим свойство 1). Зафиксируем нормализацию $A^{\prime}(0)=0$ и будем использовать обозначения (8.4). Полагая $x_{2}=0$ в (8.1), получаем

$$
C^{\prime} q_{+}^{\prime}+2 C q_{+}^{\prime \prime}=2 a_{0} q_{+}^{\prime \prime}+a_{2} q_{+}
$$

Решая при $q_{+} \neq 0$ уравнение (8.5) относительно $C$, получаем (8.3).

Для проверки свойств 2)-4) следует положить $x_{1}=0, x_{1}=x_{2}$ и $x_{1}=-x_{2}$ в (8.1). Предложение доказано.

Рассмотрим равенство (8.3). Чтобы знаменатель $\left(q^{\prime}\right)^{2}$ не создавал сингулярность, максимальное и минимальное значения функции $q$ должны быть равны $\pm \sqrt{\lambda_{+} / a_{2}}$. Следовательно, $\lambda_{+} / a_{2} \geqslant 0$ и $q_{+}^{2}-\lambda_{+} / a_{2} \leqslant 0$.

$\mathrm{C}$ другой стороны, можно считать нуль максимумом или минимумом функции $A$, поэтому далее мы предполагаем, что $a_{0} a_{2} \leqslant 0$.

Итак, мы установили, что

$$
a_{2} C=a_{2} a_{0}+a_{2}^{2} \frac{q_{+}^{2}-\lambda_{+} / a_{2}}{2\left(q^{\prime}\right)^{2}} \leqslant 0 .
$$

Этот факт не вступает в противоречие с равенством $C_{0}=0$ лишь в случае, когда

$$
a_{0}=a_{2}=C=0 \quad \text { или } \quad q_{+} \equiv 0 .
$$

Аналогично имеем следующие три условия:

$$
\begin{array}{rll}
c_{0}=c_{2}=A=0 & \text { или } & q_{-} \equiv 0, \\
d_{0}=d_{2}=B=0 & \text { или } & p_{+} \equiv 0, \\
b_{0}=b_{2}=D=0 & \text { или } & p_{-} \equiv 0 .
\end{array}
$$

Из одновременного выполнения условий (8.6)-(8.9) либо следует утверждение теоремы, либо следует, что $q_{+}=q_{-}=p_{+}=p_{-} \equiv 0$. Иными словами, при Bcex $x$

$$
A(x)=C(x)=A(-x), \quad B(x)=D(x)=B(-x)
$$


При условии (8.10) уравнение (8.1) принимает вид

$$
\begin{aligned}
\partial_{2}\left(\partial_{1}\right. & \left.+\partial_{2}\right)\left(A\left(x_{2}\right) B\left(x_{1}+x_{2}\right)\right)+\partial_{2}\left(\partial_{1}-\partial_{2}\right)\left(A\left(x_{2}\right) B\left(x_{1}-x_{2}\right)\right) \\
& =\partial_{1}\left(\partial_{1}+\partial_{2}\right)\left(A\left(x_{1}\right) B\left(x_{1}+x_{2}\right)\right)-\partial_{1}\left(\partial_{1}-\partial_{2}\right)\left(A\left(x_{1}\right) B\left(x_{1}-x_{2}\right)\right),
\end{aligned}
$$

где функции $A$ и $B$ четные, $2 \pi$-периодические и имеют нулевые средние.

Дифференцируя (8.11) по $x_{2}$ и полагая $x_{2}=0$, получаем уравнение

$$
\left(A^{\prime} B^{\prime}+2 A B^{\prime \prime}\right)^{\prime}=4 a_{2} B^{\prime}+2 a_{0} B^{\prime \prime \prime}, \quad a_{0}=A(0), \quad a_{2}=A^{\prime \prime}(0) .
$$

Отметим, что в силу четности $A$ величины $A^{\prime}(0)$ и $A^{\prime \prime \prime}(0)$ равны нулю. Решая при $B \neq$ const уравнение (8.12) относительно $A$, получаем

$$
A=a_{0}+\frac{2 a_{2} B^{2}+b B-\lambda}{\left(B^{\prime}\right)^{2}}, \quad b, \lambda=\text { const. }
$$

Остается повторить предыдущие рассуждения. Чтобы знаменатель $\left(B^{\prime}\right)^{2}$ не создавал сингулярность, необходимо иметь

$$
a_{2} \neq 0, \quad \frac{1}{a_{2}}\left(\lambda+\frac{b^{2}}{16 a_{2}^{2}}\right) \geqslant 0,
$$

так что выражение

$$
\frac{2 a_{2} B^{2}+b B-\lambda}{a_{2}\left(B^{\prime}\right)^{2}}
$$

оказывается неположительным.

Кроме того, можно считать, что $a_{0} a_{2} \leqslant 0$. Поэтому $A$ не меняет знак и с учетом равенства $A_{0}=0$ должно обратиться в нуль. Альтернативой является условие $B=$ const. Теорема доказана.

\section{Список литературы}

1. В. В. Козлов, Н. В. Денисова, "Полиномиальные интегралы геодезических потоков на двумерном торе", Матем. сб., 185:12 (1994), 49-64; англ. пер.: V. V. Kozlov, N. V. Denisova, "Polynomial integrals of geodesic flows on a two-dimensional torus", Russian Acad. Sci. Sb. Math., 83:2 (1995), 469-481.

2. Н. В. Денисова, В.В. Козлов, "Полиномиальные интегралы обратимых механических систем с конфигурационным пространством в виде двумерного тора", Матем. сб., 191:2 (2000), 43-63; англ. пер.: N. V. Denisova, V. V. Kozlov, "Polynomial integrals of reversible mechanical systems with a two-dimensional torus as the configuration space", Sb. Math., 191:2 (2000), 189-208.

3. А. Е. Миронов, "О полиномиальных интегралах механической системы на двумерном торе", Изв. РАН. Сер. матем., 74:4 (2010), 145-156; англ. пер.: А. Е. Mironov, "On polynomial integrals of a mechanical system on a two-dimensional torus", Izv. Math., 74:4 (2010), 805-817.

4. M. A. Olshanetsky, A. M. Perelomov, "Completely integrable Hamiltonian systems connected with semisimple Lie algebras", Invent. Math., 37:2 (1976), 93-108.

5. Г. Харди, Расходящиеся ряды, ИЛ, М., 1951; пер. с англ.: G. Н. Hardy, Divergent series, Clarendon Press, Oxford, 1949. 
6. В. В. Козлов, Д. В. Трещёв, "Об интегрируемости гамильтоновых систем с торическим пространством положений”, Матем. сб., 135(177):1 (1988), 119-138; англ. пер.: V. V. Kozlov, D. V. Treshchëv, "On the integrability of hamiltonian systems with toral position space", Math. USSR-Sb., 63:1 (1989), 121-139.

7. В.В. Козлов, Симметрии, топология и резонансы в гамильтоновой механике, Изд-во Удмуртского ун-та, Ижевск, 1995; англ. пер.: V. V. Kozlov, Symmetries, topology and resonances in Hamiltonian mechanics, Ergeb. Math. Grenzgeb. (3), 31, Springer-Verlag, Berlin, 1996.

8. G. Bozis, "Compatibility conditions for a nonquadratic integral of motion", Celestial Mech., 28:4 (1982), 367-380.

H. В. Денисова (N. V. Denisova)

Московский государственный университет

им. М. В. Ломоносова,

механико-математический факультет

E-mail: ndenis@mech.math.msu.su

В. В. Козлов (V. V. Kozlov)

Математический институт им. В. А. Стеклова РАН

E-mail: kozlov@pran.ru

Д. В. ТРещЁв (D. V. TReschev)

Математический институт им. В. А. Стеклова РАН

E-mail: treschev@mi.ras.ru
Поступило в редакцию 25.04.2012 